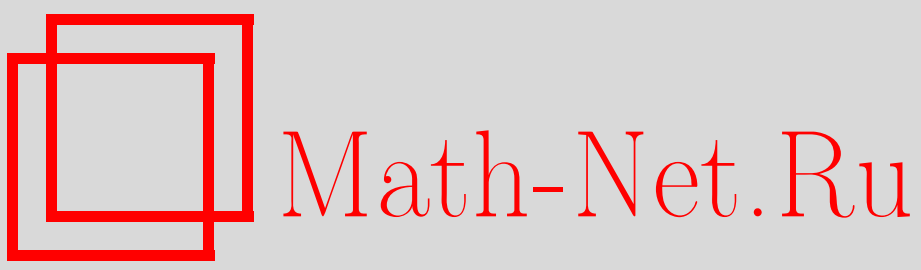

С. Ю. Немировский, Штейновы области на алгебраических многообразиях, Матем. заметки, 1996, том 60, выпуск 2, 295-298

DOI: https://doi.org/10.4213/mzm1827

Использование Общероссийского математического портала MathNet.Ru подразумевает, что вы прочитали и согласны с пользовательским соглашением

http://www. mathnet.ru/rus/agreement

Параметры загрузки:

IP : 54.172 .240 .79

26 апреля 2023 г., 15:32:25

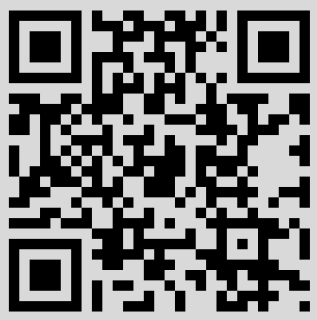




\section{ШТЕЙНОВЫ ОБЛАСТИ НА АЛГЕБРАИЧЕСКИХ МНОГООБРАЗИЯХ}

\section{С. Ю. Немировский}

0. Введение. В последние полтора десятилетия в ряде работ изучались геометрия и топология штейновых и строго псевдовыпуклых областей (см. обзор [1]). Такие области в принципе можно использовать для доказательства алгебраичности или рациональности компактных многообразий в духе классической работы Г. Грауэрта [2] (см. также [3]). Поэтому определенный интерес представляет выяснение возможного расположения штейновых областей на комплексных многообразиях.

Известно (см. [2]), что строго псевдовыпуклая область не может содержать компактных комплексных гиперповерхностей с положительным нормальным расслоением (соответственно в двумерном случае все комплексные кривые в такой области имеют отрицательный индекс самопересечения [3]). Кроме того, существует классический способ построения строго псевдовьпуклого исчерпания дополнения к подобной гиперповерхности [4]. Поскольку на компактной алгебраической поверхности "много" кривых с положительным индексом самопересечения, можно спросить, а верно ли, что любая строго псевдовыпуклая область на ней лежит в дополнении к некоторой кривой. Этот вопрос возник в [3] в связи с теоремой Ходжа об индексе для строго псевдовыпук лых областей.

В настоящей заметке мы покажем, что ответ отрицателен даже для штейновых строго псевдовыпуклых областей, по крайней мере, если нет дополнительных ограничений на поверхность. Кроме того, будет установлено, что для многообразий большей размерности ответ также отрицателен уже в простейшем случае $\mathbb{C P}^{n}$.

Сформулируем теперь точно полученные результаты. Мы будем строить области $U \subset X$ на комплексном многообразии, удовлетворяющие следующему условию $(*)$ :

любое линейное расслоение на $X$ вида $\mathscr{O}(D)$, где $D$ - эффективный дивизор, нетривиально на $U$.

Это условие заведомо не выполнено, если в $X \backslash U$ содержится компактная комплексная гиперповерхность.

Теорема 1. Можно указать рачиональную поверхность и штейнову строго псевдовыпуклую область на ней, удовлетворяющ,ую условию (*). Такие области существуют также на любой поверхности с численно неотричательным каноническим классом.

Численная неотрицательность означает, что для любой комплексной кривой $C \subset X$ индекс пересечения $\mathscr{K}_{X} \cdot C \geqslant 0$.

ТЕОРема 2. Штейновы области, удовлетворяющие условию (*), существуют в $\mathbb{C P}^{N}$ для любого $N \geqslant 3$.

Работа выполнена при поддержке Российского фонда фундаментальных исследований, грант № 93-01-00225, и Международного научного фонда, грант 508_s. 
Отметим дополнительно, что построенные области могут иметь сколь угодно малый объем. Фактически они будут окрестностями вещественных двумерных подмногообразий.

Автор благодарен Н. Кружилину за обсуждения по теме работы и А. Домрину, обратившему его внимание на работу Форстнерича [5].

1. Вещественные деформации кривых. Пусть $S \subset X$-вещественная ориентированная поверхность, вложенная в компактную комплексную поверхность. После малой деформации существует лиш конечное число комплексных точек $S$, в которых касательная плоскость к $S$ есть комплексная прямая. В зависимости от вида пересечения $S$ с касательной в некоторых локальных координатах комплексные точки подразделяются на эллиптические и гиперболические.

Обозначим через $e_{ \pm}$и $h_{ \pm}$число эллиптических и гиперболических комплексных точек, в которых ориентация касательной плоскости совпадает или не совпадает с комплексной. Введем также стандартным образом индексы $I_{ \pm}=e_{ \pm}-h_{ \pm}$.

Согласно результатам Громова и Харламова, Элиашберга (подробное "элементарное" доказательство см. у Форстнерича в [5]) если, скажем, $e_{+} h_{+}>0$, то существует изотопная $S$ вложенная поверхность $S^{\prime}$ с $e_{+}^{\prime}=e_{+}-1$ и $h_{+}^{\prime}=h_{+}-1$, т.е. возможно "взаимоуничтожение" комплексных точек различного типа с одинаковой ориентацией касательной плоскости.

По формулам Лая [6]

$$
I_{+}+I_{-}=\chi(S)+S^{2}, \quad I_{+}-I_{-}=c_{1}(X) \cdot S
$$

где $\chi(S)$ - эйлерова характеристика $S, S^{2}$ - индекс самопересечения, а $c_{1}(X)$ первый класс Черна $X$.

Если $S$ гомологична неособой комплексной кривой того же рода, то по формуле присоединения $c_{1}(X) \cdot S=\chi(S)+S^{2}$, т.е. $I_{-}=0$, а $I_{+}=c_{1}(X) \cdot S$.

В этой ситуации мы видим, что справедлива

Лемма 1.1. Пусть $S$-неособая комплексная кривая на $X$ с $c_{1}(X) \cdot S \leqslant 0$. Тогда существует изотопная $S$ влохсенная вещественная поверхность, имеющая только гиперболические комплекснье точки с комплексной ориентаиией.

2. Построение штейновых областей. Небольшая модификация рассуждения из [5, предложение 6.1] дает следующий способ построения штейновых областей на комплексных поверхностях.

ЛЕмма 2.1. Пусть $S$ - влохсенная вещественная поверхность такая, что $e_{ \pm}=0$. Тогда существует базис штейновых строго псевдовыпуклых окрестностей $S$.

Комбинируя эту лемму с леммой 1.1 , мы получаем основной технический результат.

ЛЕмма 2.2. Пусть $S$ - неособая комплексная кривая на комплексной поверхности $X$, и $c_{1}(X) \cdot S \leqslant 0$. Тогда на $X$ существует штейнова область $U$ такая, что ограничение на нее любого линейного расслоения вида $\mathscr{O}(D)$ c $D \cdot S>0$ нетривиально. В частности, если $D \cdot S>0$ для любой кривой $D$, то $U$ удовлетворяет условию $(*)$.

Теперь мы можем доказать сформулированные во введении теоремы. 
ДоКАЗАТЕЛЬСТВо ТЕОРЕМЫ 1. Сначала построим штейнову область, удовлетворяющую $(*)$, на рациональной поверхности.

Пусть $S_{0}$ - гладкая квартика в $\mathbb{C P}^{2}$. Тогда род $g\left(S_{0}\right)=3$, и $S_{0}^{2}=16$. Пусть $H \subset S$ - дивизор гиперплоского сечения, а $P_{0} \in S$ - фиксированная точка.

Выберем 12 различных точек $x_{j}$ на $S_{0}$ так, чтобы точки на якобиане $\mathscr{J}\left(S_{0}\right)$, соответствуюшие линейным расслоениям $\mathscr{O}\left(x_{j}-P_{0}\right), j=1, \ldots, 12$, и $\mathscr{O}\left(H-4 P_{0}\right)$, были линейно независимы над $\mathbb{Z}$. В частности, это означает, что не существует кривой $C \subset \mathbb{C P}^{2}$, пересекающейся с $S_{0}$ только в точках $x_{j}$. Действительно, в противном случае имела бы место линейная эквивалентность

$$
\sum_{j=1}^{12} n_{j} x_{j} \cong \operatorname{deg} C \cdot H, \quad n_{j} \in \mathbb{Z}
$$

что противоречит выбору точек $x_{j}$.

Раздуем теперь $\mathbb{C P}^{2}$ в точках $x_{j}$ и обозначим полученную поверхность через $X$, а собственный прообраз $S_{0}$ - через $S$. Тогда $S^{2}=S_{0}^{2}-12=4, \chi(S)=\chi\left(S_{0}\right)=$ $2-2 g\left(S_{0}\right)=-4$, и любая кривая на $X$ пересекается с $S$ (иначе образ этой кривой в $\mathbb{C P}^{2}$ пересекал бы $S_{0}$ только в точках $x_{j}$ ).

Остается применить к $X$ и $S$ лемму 2.2 .

Отметим, что эта конструкция аналогична построению Х. Хиронаки кривой рода 1 на рациональной поверхности, стягиваемой по Грауэрту в неалгебраическую точку.

Теперь рассмотрим случай поверхности $X$ с численно неотрицательным каноническим классом. Выберем в качестве кривой $S$ общее гиперплоское сечение, тогда $S$ неособа по теореме Бертини, и индекс пересечения $S$ с любой кривой положителен. По предположению $c_{1}(X) \cdot S=-\mathscr{K}_{X} \cdot S \leqslant 0$, и мы снова оказываемся в ситуации леммы 2.2 , что и требовалось доказать.

Построенные штейновы области весьма далеки от того, чтобы лежать в аффинной части поверхности; напротив, они являются деформациями окрестности гиперплоского сечения.

ДОКАЗАТЕЛЬСТВО ТЕОРЕМЫ 2. Рассмотрим К3-поверхность $X$, вложенную в $\mathbb{C P}^{3}$ как гиперповерхность степени 4 . На $X$ существует гладкая рациональная кривая $S$ с индексом самопересечения -2 . (Существование такой кривой следует из формулы Римана-Роха и формулы для рода, см. [7, гл. VIII].) Поскольку $c_{1}(X)=0$, из леммы 2.2 получаем штейнову область $U \subset X$ такую, что на ней нетривиально любое положительное линейное расслоение на $X$.

Пусть теперь $V$ - такая область в $\mathbb{C P}^{N} \supseteq \mathbb{C P}^{3}$, что $V \bigcap X=U$. Применяя к паре $U \subset V$ теорему Сью [8] о существовании штейновой окрестности штейнова подмногообразия, получаем, что найдется штейнова область в $\mathbb{C P}^{N}$, на которой нетривиально гиперплоское расслоение, что и требовалось доказать.

Конечно, можно было бы взять в качестве $X$ произвольную гладкую гиперповерхность в $\mathbb{C P}^{N}$ степени $d \geqslant 4$. В этом случае канонический класс $X$ равен $(d-4) H$ и неотрицателен, значит, по теореме 1 существует нужная для построения область $U \subset X$. Мы привели несколько более сложный пример, поскольку полученная в нем штейнова область имеет простейший возможный гомотопический тип двумерной сферы. 
В заключение отметим, что построения, использующие лемму 2.2 , невозможны на поверхностях с отрицательньм каноническим классом, например, на $\mathbb{C P}^{2}$. Поэтому для них вопрос о существовании удовлетворяющих условию $(*)$ штейновых областей остается открытым. Сформулируем его для $\mathbb{C P}^{2}$ в совсем простом виде, используя тот факт, что линейное расслоение на двумерном штейновом многообразии полностью определяется своим вещественным первым классом Черна.

ВопРос. Верно ли, что ограничение формы Фубини-Штуди на строго псевдовыпуклую область в $\mathbb{C P}^{2}$ когомологично нулю?

Именно для этого случая в [3] было предложено применение к одной задаче А. Г. Витушкина.

Московский государственный университет им. М.В. Ломоносова

Поступило 01.03 .96

\section{СПИСОК ЦИТИРОВАННОЙ ЛИТЕРАТУРЫ}

1. Bennequin D. // Asterisque. 1990. №189-190. P. 285-323. 2. Грауэрт $Г$. // Сб. "Комплексные пространства". М.: Мир, 1965. Р. 205-299. 3. Немировский С. Ю. // Матем. заметки. 1996. V. 60. № 3. 4. Suzuki O. // Publ. Res. Inst. Math. Sci. Ser. A. 1975. V. 11. №1. P. 185-199. 5. Forstneric F. // Duke Math. J. 1992. V. 67. № 2. P. 353-376. 6. Lai H. F. // Trans. Amer. Math. Soc. 1972. V. 172. P. 1-33. 7. Barth W., Peters C., Van de Ven A. Compact complex surfaces. N. Y., 1984. 8. Siu Y.-T. // Invent. Math. 1976. V. 38. № 1. P. 89-100. 